\title{
Pigmented villonodular synovitis
}

\author{
Uri Givon, Abraham Ganel, Michael Heim
}

\begin{abstract}
Pigmented villonodular synovitis (PVS) is an uncommon, usually monoarticular disorder encountered mainly in adults. A boy and a girl, both 7 years old, were referred because of recurrent knee effusions. Both were medically treated for other rheumatic disorders for five years. PVS was diagnosed by arthroscopy and synovectomy was curative in both cases.
\end{abstract}

Pigmented villonodular synovitis (PVS) comprises a group of idiopathic lesions in joints, tendon sheathes, and bursae. The clinical presentation is of recurrent joint effusions, which can be misdiagnosed as arthritis. Episodes of complete remission may be found between periods of active disease. The histological findings are exuberant synovial proliferation with numerous villi and folds that sometimes fuse into nodules and form locally aggressive intra-articular masses. ${ }^{12}$ PVS is usually found in young adults and only a few cases were previously reported in children. Because of its rarity diagnosis of PVS in children may be made late. We report two cases of children who were medically treated for suspected rheumatic disorders suffering adverse side effects until the correct diagnosis was made.

\section{Case reports}

CASE 1

A previously healthy 2 year old girl started to suffer from recurrent effusions of her left knee. The parents were of Iraqi and Turkish Jewish origin. Juvenile rheumatoid arthritis or familial Mediterranean fever were suspected. Treatment by non-steroidal anti-inflammatory drugs and colchicine was initiated and prednisone and penicillamine were added later. Adverse side effects such as vomiting, diarrhoea, and abdominal pain were encountered, however remissions occurred independently of treatment. The girl was seen in our clinic at the age of 7 years. On examination no abnormalities were found other than in the left knee, which was swollen and warm with limited range of movement. Serological tests and concentrations of antinuclear antibodies were normal; normal results were found on radiography. Arthroscopy demonstrated hypertrophic synovium and defects in the articular cartilage. PVS was diagnosed by a synovial biopsy specimen (figure) and radical synovectomy was curative. Three years later the girl was asymptomatic and no recurrence was found.

CASE 2

(Arch Dis Child 1991;66:1449-50) A boy of European Jewish origin had had

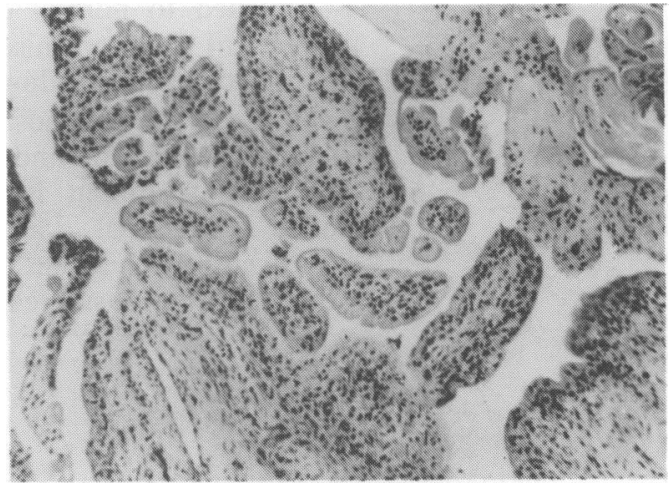

Biopsy specimen of case 1 showing villous hypertrophy, diffuse histiocytic proliferation, and focal haemosiderin deposition (haemotoxylin and eosin $\times 100$ ).

recurrent right knee effusions since the age of 2 years. He was completely healthy between the spells of the disease. A diagnosis of familial Mediterranean fever had been made and nonsteroidal anti-inflammatory drugs and colchicine were prescribed but discontinued because of vomiting and diarrhoea. At the age of 7 years the frequency of attacks increased. On examination the right knee was swollen with limited range of movement. Aspiration yielded serosanguineous fluid. Serological tests and concentrations of antinuclear antibodies were normal; normal results were found on radiography. Arthroscopy demonstrated a large bleeding nodular mass that was excised and on histological examination the diagnosis of PVS was made. The boy was completely healthy one year later without any signs of recurrence.

\section{Discussion}

PVS was first described by Jaffe et al in 1941. Most of the cases were described in young adults. ${ }^{1-3}$ We were able to locate seven reports of PVS in children. Bobechko and Kostuik reported three cases of children with recurrent knee effusions and cutaneous haemangiomas over the involved knees who were found to have PVS. ${ }^{3}$ Wendt et al reported three generations of the same family who suffered from PVS and multiple lentigines syndrome-a rare skin disease. Two siblings from the third generation had multiple joint involvement. ${ }^{4}$ More cases were described in children who had other diseases as well. ${ }^{5}{ }^{6}$ As far as we could ascertain our patients are the first healthy children found to have PVS.

Diagnosis of PVS is usually made late, especially if the afflicted joint is a deep one, such as the hip or shoulder joint. ${ }^{1}$ Further delay may be attributed in our cases to the relatively high prevalence of familial Mediterranean fever, a similar joint disorder, in the Israeli popula- 
tion. This disease is hard to rule out and long term medical treatment is indicated in order to prevent late complications.

Treatment of PVS is surgical and $67 \%$ of the patients respond well to a radical synovectomy. In $33 \%$ of the cases a recurrence was reported and in $22 \%$ the result was unsatisfactory. Our patients both had a very good result and returned to normal childhood activities after years of a compromised quality of life.

In conclusion, though rare, PVS occurs in children. Misdiagnosis is associated with prolonged morbidity and various adverse effects of medical treatment. PVS should be considered in the differential diagnosis of recurrent joint effusion in children. Early arthroscopy should perhaps be considered in cases of pauciarticular arthritis when antinuclear antibodies are not present.

1 Schiller AL. Tumors and tumor like lesions involving joints In: Kelley WN, Harris ED, Ruddy S, Sledge CB, eds Textbook of rheumatology. Philadelphia: WB Saunders, 1989:1783-9.

2 Jaffe HL, Lichtenstein N, Sutro CJ. Pigmented villonodular synovitis, bursitis and tenosynovitis. Archives of Pathology 1941;31:731-65.

3 Bobechko WP, Kostuik JP. Childhood villonodular synovitis. Can F Surg 1968;11:480-6.

4 Wendt RG, Wolfe F, McQueen D, Murphy P, Solomon H Housholder $M$. Polyarticular pigmented villonodular synovitis in children: evidence for genetic contribution F Reumatol 1986;13:921-6.

Walls JP, Nogi J. Multifocal pigmented villonodular synovitis in a child. F Pediatr Orthop 1985;5:229-31.

6 Leszczynski JR, Huckell JS, Percy JC. Pigmented villonodular synovitis in multiple joints, occurrence in a child with cavernous haemangioma of the lip and pulmonary stenosis. Ann Rheum Dis 1975;34:269-72. 